\title{
Kosovokrieg, Kriegsberichterstattung und die Popularität der deutschen Regierungsparteien und -politiker
}

\author{
Evelyn Bytzek*
}

Am Beispiel des Kosovokriegs 1999 wird auf die Frage nach den Wirkungen von Kriegen auf die Popularität der deutschen Regierung eingegangen, insbesondere auf das mögliche Auftreten einer "Rally" (von "Rally round the flag", dem Scharen um die Flagge). Hierbei wird davon ausgegangen, dass eine Rally durch eine für die Regierung positive Medienberichterstattung aufgrund des Informationsmonopols der Regierung bei RallyEreignissen zustande kommt. Mit Hilfe einer thematischen Inhaltsanalyse der Tageszeitungen FAZ und SZ werden Hypothesen über die Wirkung des Kosovokriegs zu verschiedenen Zeitpunkten und für verschiedene Teile der Wählerschaft formuliert. Anhand einer Faktorenanalyse auf Basis von aus Umfragen gewonnenen Skalometerwerten zu deutschen Parteien und Politikern lässt sich nachweisen, dass die Popularität der Regierungsakteure im ersten Kriegsmonat, dem April 1999, aufgrund des Vorberrschens von für die Regierung positiven Themen in den Medien anstieg, danach jedoch durch das Aufkommen von für die Regierung negativ besetzten Themen sank und nach dem Krieg im Juni 1999 für die meisten Regierungsakteure auf das Vorkriegsniveau zurückkebrte, so dass man nur für den ersten Kriegsmonat von einer Rally sprechen kann.

Keywords: Rally, Krieg, Informationsmonopol, Regierungsmitglieder, Popularität, Inhaltsanalyse

\section{Einleitung}

Die häufige Beobachtung, dass internationale Krisen und allen voran Kriege die Popularität von Regierungen steigern können, führte zu einer regen Forschungstätigkeit zu diesem Phänomen, das unter dem Namen „Rally“ (von „Rally round the flag“, dem Scharen um die Flagge) bekannt ist. Für den deutschen Fall liegen jedoch kaum Befunde vor, da Deutschland nach dem Zweiten Weltkrieg nicht direkt an Kampfhandlungen beteiligt war. Der Kosovokrieg im Frühjahr 1999, an dem deutsche Soldaten im Rahmen des NATO-Einsatzes mitwirkten, änderte diese Situation. Er bietet somit die Möglichkeit, der Frage nachzugehen, ob auch in Deutschland eine Rally auftreten kann, ob also die deutsche Regierung vom Kosovokrieg insofern profitieren konnte, als dass ihre Unterstützung in der deutschen Bevölkerung durch den Krieg stieg. Durch die Übertragung der Forschung zum Zusammenhang zwischen Kriegen und Regierungspopularität auf den deutschen Fall lassen sich Einblicke auf die Wirkung von Kriegen auf Koalitionsregierungen gewinnen, ein bislang wenig beleuchtetes Feld. Daher sollen im Folgenden die Auswirkungen des Kosovokriegs auf die Popularität der deutschen Regierungsparteien und der hauptsächlich betroffenen Politiker - Bundeskanzler, Außenund Verteidigungsminister - im Rahmen des deutschen Parteiensystems untersucht

* Hiermit bedanke ich mich ganz herzlich bei Prof. Dr. Franz Urban Pappi, Dr. Susumu Shikano und Dominik Steegmüller für ihre Hilfe. 
werden. Hierfür wird im zweiten Abschnitt zunächst das Rally-Phänomen beleuchtet und darauf eingegangen, durch welche Mechanismen eine Rally in Gang kommt. Wesentlich für das Entstehen einer Rally ist laut Brody, dass die Darstellung der Regierung in den Medien in Bezug auf das Rally-Ereignis aufgrund ihres Informationsmonopols positiv ist (1991: 64). Des Weiteren wird der Frage nachgegangen, welche Teile der Wählerschaft eine Rally tragen. Da die Medienberichterstattung beim Zusammenhang zwischen Krieg und Regierungspopularität eine wesentliche Rolle spielt, beschäftigt sich der dritte Abschnitt mit dem Bild des Kosovokriegs in den Medien. Zusammen mit den theoretischen Überlegungen aus Abschnitt 2 werden anhand des Medienbilds Hypothesen zur Wirkung des Kosovokriegs auf die Popularität der deutschen Regierungsakteure formuliert, die im vierten Abschnitt anhand von Umfragedaten empirisch überprüft werden.

\section{Das Rally-Phänomen und Untersuchungsplan}

\subsection{Wie kommt eine Rally in Gang?}

Das Phänomen einer „Rally round the Flag“, dem Scharen einer Nation um ihre Flagge angesichts einer internationalen Krise, in die die USA verwickelt sind, ist seit Muellers Studie von 1970 zu den Determinanten der Popularität des US-Präsidenten eine prominente Erklärung für das sprunghafte Ansteigen an Unterstützung für den Präsidenten. ${ }^{1}$ Die wahrgenommene Bedrohung der Nation von außen zieht dabei einen engeren $\mathrm{Zu}-$ sammenhalt der Bürger des Landes nach sich, der über den gestiegenen Patriotismus auch eine stärkere Unterstützung der nationalen Führerschaft mit sich bringt (McLeod u. a., 1994: 21). An dieser einfachen Patriotismus-Erklärung für das Auftreten einer Rally wurde mehrfach Kritik laut. So weist Brody darauf hin, dass nicht jede internationale Krise, die laut Mueller eine Rally zur Folge haben sollte, dies auch tut (1991: 62). Daher sucht Brody die Erklärung für eine Rally nicht in einer reflexartigen patriotischen Antwort auf eine äußere Bedrohung, sondern in den speziellen Dynamiken, die bei einem solchen Ereignis in der Regel auftreten. Da sich ein Rally-Ereignis schnell entwickelt, besitzt die Regierung gegenüber ihren Kritikern ein Informationsmonopol. Die Medien können daher ausschließlich die Informationen der Regierung, die deren Standpunkt vertreten, nutzen, wodurch eine steigende Unterstützung durch die Bevölkerung zustande kommt (Brody, 1991: 64).

Diese Medien-Hypothese Brodys zur Entstehung einer Rally beinhaltet einen für die Wirkung von Kriegen wichtigen Aspekt: die Medienberichterstattung über den Krieg. Die für den amerikanischen Fall untersuchten Kriege fanden nicht auf amerikanischem Boden statt und waren damit für den größten Teil der Bevölkerung nicht direkt, sondern nur über die Medienberichterstattung erfahrbar. Gleiches trifft auf den Kosovokrieg und die deutsche Bevölkerung zu. Daher kann die Medienberichterstattung we-

1 Kriege werden von Mueller jedoch zuerst als negativer Einfluss auf die präsidentielle Popularität modelliert, als Beispiel dienen der Korea- und der Vietnamkrieg (Mueller, 1970: 23). In späteren Arbeiten stellt er jedoch fest, dass Kriege anfangs auch zu einem Anstieg der Popularität des Präsidenten führen und damit zu den Rally-Ereignissen gezählt werden sollten (Mueller, 1973: 58). Dieser Befund wurde von etlichen Untersuchungen bestätigt, so dass sich das Einordnen von Kriegen, zumindest zu deren Beginn, unter die Rally-Ereignisse durchgesetzt hat (vgl. hierzu Norpoth, 1988). 
sentlichen Einfluss auf die Wirkung von Kriegen nehmen. Hierbei lässt sich eine Vielzahl von Einflussmöglichkeiten der Medienberichterstattung auf die Bewertung der Rezipienten denken. In der jüngeren Medienwirkungsforschung wird vor allem die Macht der Medien belegt, allein durch die Lenkung von Aufmerksamkeit, d. h. durch die unterschiedliche Betonung von Themen oder subthematischen Teilaspekten, die Beurteilung politischer Sachverhalte zu beeinflussen (Schenk, 2002: 296-303 und 710). In diesem Zusammenhang werden vor allem zwei Wirkungsmechanismen diskutiert: MedienPriming und Medien-Framing. Medien-Priming bedeutet, dass Nachrichten die Kriterien, anhand derer die politische Führungsmannschaft bewertet wird, allein durch die unterschiedliche Betonung verschiedener Themen beeinflussen. Ein Thema, das prominent in den Medien vertreten ist, wird zur politischen Bewertungsgrundlage, wenn Bewertungen der Politiker und Parteien zu diesem Thema maßgeblich für deren Gesamtbewertungen werden (Iyengar/Simon, 1993: 368). Medien-Framing hingegen „... ist ein Vorgang, bei dem (1) bestimmte Objekte und Relationen zwischen Objekten betont, also bestimmte Ausschnitte der Realität beleuchtet werden und (2) bestimmte Maßst $\ddot{a}$ be bzw. Attribute, die man an Objekte anlegen kann, salient gemacht werden“ (Scheufele, 2003: 46). Die Medien heben also innerhalb eines Sachverhalts, in diesem Fall bei der Darstellung eines Krieges, bestimmte Aspekte hervor, die positiv oder negativ für die Regierung sein können, und legen dem Rezipienten so eine bestimmte Bewertung der Regierung hinsichtlich dieses Sachverhalts nahe. Gerade bei bislang unbekannten Sachverhalten, wie eben dem Kampfeinsatz Deutschlands im Kosovokrieg, können beide Prozesse zusammenwirken, indem dem Rezipienten durch das Framing eine bestimmte Bewertung der Regierung in Bezug auf den Kosovokrieg nahe gelegt wird und diese Bewertung durch das Priming wesentlich für die Gesamtbewertung der Regierung durch den Rezipienten wird.

Iyengar und Simon untersuchten den Effekt des Medien-Priming auf die öffentliche Meinung am Fall des Golfkriegs 1991. Ein wesentliches Ergebnis ihrer Studie lautet, dass eine bessere außenpolitische Bewertung zur Zeit des Golfkriegs zu einer besseren Gesamtbewertung des Präsidenten führte, im Gegensatz zur Zeit vor dem Golfkrieg, in der ökonomische Bewertungen für die Gesamtbewertung wesentlich waren (Iyengar/Simon, 1993: 376). Dies bedeutete eine Bestätigung für die Priming-Hypothese, da durch die außerordentliche Medienpräsenz des Golfkriegs das Thema Außenpolitik in den Vordergrund rückte. Allen u. a. (1994) stellten ebenfalls für den Golfkrieg 1991 fest, dass es nach einem ersten Anstieg der Unterstützung bei Kriegsbeginn Mitte Januar $1991 \mathrm{zu}$ einem zweiten Anstieg Ende Februar 1991 mit Beginn der Bodenoffensive kam, der nicht durch die Rally-Hypothese erklärt werden kann. Hier sehen sie eine Schweigespirale am Werk, die durch das für den Golfkrieg geprägte Meinungsklima anderslautende Meinungen durch Selbstzensur unterdrückte und so die Mehrheitssicht immer weiter stärkte. Die Prozesse, durch die eine Schweigespirale in der öffentlichen Meinung auftreten kann, sind Allen u. a. zufolge das Medien-Priming und -Framing. Die kontinuierliche und extensive Berichterstattung über den Golfkrieg lässt sie davon ausgehen, dass die Meinungen der Rezipienten zu diesem Ereignis zur Bewertungsgrundlage werden können, also ein Priming-Effekt eintritt. Anhand exemplarischer Fälle der Medienberichterstattung zeigen Allen u. a., dass negative Sichtweisen durch das negative Framing von Antikriegsmeinungen und -handlungen, wie z. B. Demonstrationen, und das positive Framing der unterstützenden Äußerungen als patriotisch zusammen mit dem Framing des Golfkriegs in weitgehend technisch-militärischen Begriffen unterdrückt werden. Eine hierdurch in Gang gesetzte Schweigespirale führte dann zu einem zweiten Anstieg der Unterstützung für den Golfkrieg (Allen u. a., 1994: 271ff.). Der erste An- 
stieg an Unterstützung für den Golfkrieg zu Kriegsbeginn wird hingegen auf eine Rally zurückgeführt (Allen u. a., 1994: 269).

Diese Schlussfolgerung ist insofern problematisch, als sie die Mechanismen, die die erste Rally bewirkten, nicht beleuchten. Dabei erscheint es aufgrund der vorgestellten Forschungsergebnisse sinnvoll, die Mechanismen, die eine Schweigespirale bewirken können, auch zur Erklärung der Rally an sich heranzuziehen. Ein Modell des Rally-Phänomens würde dann wie folgt aussehen: Zu Beginn einer internationalen Krise oder eines Krieges befindet sich die Regierung in der Lage, durch ihr Informationsmonopol die Medienberichterstattung, die auf ihre Informationen angewiesen ist, für sich positiv zu gestalten. Diese für die Regierung günstige Berichterstattung wirkt im Gegenzug auf die Meinungen der Bürger durch Medien-Priming und -Framing. Durch eine außerordentliche Berichterstattung über den Krieg treten andere Themen als Bewertungsbasis in den Hintergrund. Das Framing der Berichterstattung in für die Regierung positive Themenaspekte bewirkt wiederum eine positive Bewertung der Regierung im geprimten Thema, dem Krieg. Die Voraussetzungen für das Auftreten einer Rally sind daher die prominente Berichterstattung über den Krieg und das Hervorheben für die Regierung positiver Themenaspekte.

\subsection{Wer trägt die Rally?}

Neben der Frage zum Zustandekommen einer Rally stellt sich auch die Frage, ob der Anstieg an Unterstützung für die politische Führungsmannschaft während einer internationalen Krise von der gesamten Wählerschaft oder nur von Teilen dieser getragen wird. Sigelman und Conover untersuchten in diesem Zusammenhang den Einfluss der Parteiidentifikation der Wähler als wesentliche Determinante der Popularität des Präsidenten auf die Dauer und Reichweite einer Rally (1981: 306f.). Anhand der Geiselnahme amerikanischer Botschaftsangehöriger im Iran 1979/80 konnten sie zeigen, dass nur kurz nach Ausbruch der Krise die Rally die Parteiidentifikation als Hauptdeterminante der Unterstützung weitgehend aushebeln konnte, indem sowohl Anhänger der demokratischen als auch der republikanischen Partei und die Unabhängigen den demokratischen Präsidenten Carter zu jeweils etwa $90 \%$ unterstützten. Im Verlauf der Krise fielen die Anhänger der gegnerischen Parteien jedoch früh wieder von der Regierung ab (Sigelman/Conover, 1981: 314ff.).

Im Gegensatz dazu konstatieren Callaghan und Virtanen (1993), dass die Rally auch zu Beginn nicht von allen Wählern gleichermaßen getragen wird. Der Anstieg an Unterstützung für den Präsidenten sollte bei der Gruppe der Unabhängigen am stärksten sein, da sie hierin nicht durch parteiliche Bindungen beschränkt werden. Bei den Anhängern der Partei des Präsidenten sollte der Anstieg relativ gering sein, da die Unterstützung bereits generell höher ist als in anderen Wählergruppen. Auch bei den Anhängern der gegnerischen Partei, bei denen durch die parteiliche Bindung eine Unterstützung des Präsidenten erschwert wird, sollte der Präsident durch eine internationale Krise nicht übermäßig an Popularität gewinnen (Callaghan/Virtanen, 1993: 757). Am Beispiel der Camp David-Friedensverhandlungen 1978 und der Geiselnahme im Iran 1979/80 konnten sie die Vermutung hinsichtlich der Unabhängigen bestätigen, der Anstieg an Unterstützung war jedoch bei den Anhängern der republikanischen Partei ähnlich hoch, sank jedoch auch schnell wieder - im Gegensatz zu den Unabhängigen, die ihre Unterstützung graduell entzogen (Callaghan/Virtanen, 1993: 759f.).

Aus den beiden Untersuchungen lässt sich daher schließen, dass eine Rally nicht homogen von allen Teilen der Wählerschaft getragen wird. Die Gruppe der Unabhängigen 
scheint das größte Reservoir an Unterstützung für den Präsidenten im Falle einer internationalen Krise zu bilden, während sich die Anhänger der gegnerischen Partei nur kurz zugunsten des Präsidenten beeinflussen lassen. Problematisch an beiden Studien ist jedoch, dass sie nicht auf den Mechanismus eingehen, durch den eine Rally entsteht, also die für eine Rally notwendige prominente und für die Regierung positive Medienberichterstattung nicht beachten. Die Erklärung der unterschiedlichen Rally-Effekte ist daher unvollständig. So werden zwar die Unterschiede hinsichtlich des beschränkten Unterstützungspotenzials aufgrund von Parteibindungen zwischen den verschiedenen Wählergruppen als Grund für das schnelle Absinken der Popularität des Präsidenten bei den Anhängern der gegnerischen Partei genannt. Vor diesem Hintergrund lässt sich aber nicht erklären, warum in dieser Gruppe zu Beginn der Krise ähnliche Unterstützungszuwächse zu verzeichnen sind wie bei den Anhängern der Präsidentenpartei und den Unabhängigen. Die Erklärung von Unterschieden im Rally-Effekt zwischen Wählergruppen muss daher an den generellen Mechanismen, durch die eine Rally zustande kommt, also an Medien-Priming und -Framing, anknüpfen. Dabei erscheint es sinnvoll anzunehmen, dass verschiedene Wählergruppen nicht die gesamte Medienberichterstattung wahrnehmen, sondern jeweils nur die Medien nutzen, die normalerweise mit den Meinungen der Rezipienten konsonante Informationen liefern (Donsbach, 1989: 402). $\mathrm{Zu}$ Beginn einer internationalen Krise werden die Medien jedoch durch das Informationsmonopol der Regierung konsonant regierungsfreundlich über die Krise berichten, so dass über die Prozesse des Medien-Priming und -Framing eine Rally der gesamten Wählerschaft zu erwarten ist. Mit zunehmender Dauer der Krise werden die Informationen dann zahlreicher und auch gegnerische Stimmen laut. Diese Stimmen werden am ehesten in den Medien vertreten sein, die die Gruppe der Regierungsgegner bedienen. Die Anhänger der gegnerischen Partei werden daher zuerst mit Informationen konfrontiert, die gegen eine Unterstützung der Regierung sprechen, weshalb die Popularität der Regierung in ihren Reihen rasch sinkt. Medien, die die Anhänger der Regierungspartei bedienen, werden die Krise dagegen länger in für die Regierung vorwiegend positiven Frames darstellen, weshalb die Unterstützung durch die Anhänger der Regierungspartei nur sehr langsam sinkt. Von den Unabhängigen wird angenommen, dass sie sowohl regierungsfreundliche als auch -feindliche Informationen erhalten und daher mit ihrem Rückgang der Unterstützung zwischen den Anhängern der Regierungspartei und den Anhängern der gegnerischen Partei liegen.

\subsection{Untersuchungsplan}

Die genannten Studien beschäftigen sich ausschließlich mit dem Fall der USA und betrachten in erster Linie die durch eine Rally gestiegene Popularität des US-Präsidenten. Der vorliegende Beitrag stellt jedoch die Frage, ob eine Rally auch in der Bundesrepublik Deutschland möglich ist, und zieht dafür den Fall des Kosovokriegs 1999 heran. Es ist daher zu klären, welche politischen Akteure im deutschen Fall betrachtet werden sollen, um Anknüpfungspunkte an die Befunde der USA zu bieten, und ob der Kosovokrieg überhaupt zu den Rally-Ereignissen gezählt werden kann.

Zum Fall des Kosovokriegs ist zu sagen, dass er für Deutschland keine äußere Bedrohung darstellte. Dies ist aber für eine Rally auch nicht notwendig, zieht man die Medien-Hypothese Brodys zur Erklärung heran. Problematischer ist, dass sich der Krieg schon ab Herbst 1998 anbahnte, also fraglich ist, ob es überhaupt eine Dynamik gab, die über die Plötzlichkeit der Krise und des daraus folgenden Informationsmonopols der Regierung zu einer regierungsfreundlichen Berichterstattung über den Krieg führte. 
Diese Frage kann nur empirisch anhand einer Inhaltsanalyse beantwortet werden. Bei der Auswahl der zu betrachtenden politischen Akteure sollen nicht nur der Bundeskanzler als annäherndes Äquivalent zum US-Präsidenten, sondern alle für den Fall des Kosovokriegs relevanten politischen Akteure betrachtet werden, also die Regierungsparteien SPD und Bündnis 90/Die Grünen sowie Bundeskanzler Schröder, Verteidigungsminister Scharping und Außenminister Fischer, da für den amerikanischen Fall Befunde vorliegen, dass eine Rally nicht nur dem Chef der Exekutive zugute kommen kann (vgl. hierzu Brewer u. a., 2003: 248). Darüber hinaus sind so auch Einblicke in die Wirkungen von Kriegen auf Koalitionsregierungen möglich.

Die Analyse erstreckt sich auf den Zeitraum von März bis Juni 1999, April und Mai deckten dabei den Zeitraum des eigentlichen Kosovokriegs ab. Die Popularität der Regierungsparteien und -politiker wird anhand von Umfragedaten ermittelt. Hierfür werden die Politbarometerdaten, erhoben von der Forschungsgruppe Wahlen e.V., Mannheim, genutzt. ${ }^{2} \mathrm{Da}$ die Wirkung der Kriegsberichterstattung untersucht werden soll, ist eine enge zeitliche Bindung zwischen Umfragezeitpunkten und analysierter Berichterstattung notwendig. Dabei ist einerseits zu beachten, dass der Effekt des Medien-Priming nur dann auftritt, wenn das Priming zeitlich kurz zurückliegt, weshalb die Betrachtung der Medienberichterstattung kurz vor den Umfragen erstrebenswert ist (vgl. Peter, 2002). Andererseits soll die Analyse jedoch nicht in einzelne Befragungstage atomisiert werden, den Befragten eines Monats müssen daher die gleichen Informationen und damit die gleichen von den Medien angewandten Frames zur Verfügung stehen. Deshalb wird für die Inhaltsanalyse jeweils die Woche vor den Umfragewochen betrachtet. Es wurde jedoch sichergestellt, dass keine wesentlichen Ereignisse zum Kosovokrieg, die die Themenauswahl und damit das Framing der Medien beeinflussen und so zu unterschiedlichen Informationsbasen der Befragten eines Monats führen könnten, innerhalb der Befragungszeiträume liegen (vgl. hierfür Schütt, 2000). Es ist daher davon auszugehen, dass die Medienberichterstattung der Befragungsvorwoche das Bild widerspiegelt, das die Befragten vom Kosovokrieg im Kopf haben. Die Zeiträume der Inhaltsanalyse und Umfragen sind Tabelle $1 \mathrm{zu}$ entnehmen. Die Inhaltsanalyse beschränkt sich dabei auf die Politikteile von FAZ und SZ, da diese Medien die Funktion von Meinungsführern innerhalb des Mediensystems innehaben (Kepplinger u. a., 1986: 267).

Mit dem genauen Vorgehen bei der Analyse der Medienberichterstattung, den Ergebnissen dieser und den daraus im Zusammenhang mit der theoretischen Argumentation abgeleiteten Hypothesen zu den Wirkungen des Kosovokriegs auf die Popularität der deutschen Regierungsparteien und -politiker beschäftigt sich der dritte Abschnitt. Die Methode zur Extrahierung von Popularitätsveränderungen auf Basis von Skalometerwerten und die daraus gewonnenen Ergebnisse werden im vierten Abschnitt vorgestellt.

\section{Die Medienberichterstattung über den Kosovokrieg und Hypothesen}

Die Inhaltsanalyse der Medienberichterstattung über den Kosovokrieg soll folgende Fragen beantworten: 1) Hat der Kosovokrieg eine genügend große Medienpräsenz, so dass Priming-Effekte wahrscheinlich sind? 2) Wird der Krieg in den Medien regierungsfreundlich dargestellt, so dass eine Rally durch die gesamte Wählerschaft eintritt, oder gibt es Unterschiede in der Darstellung zwischen verschiedenen Medien, die darauf

2 Die Daten sind unter den Bezeichnungen S3261 (Westdeutschland) und S3262 (Ostdeutschland) beim Zentralarchiv für Empirische Sozialforschung der Universität zu Köln erhältlich. 
Tabelle 1: Zeiträume der Inbaltsanalyse und Umfragen

\begin{tabular}{lll}
\hline Monat & Zeitraum der Inhaltsanalyse & Umfragezeitraum \\
\hline März 1999 & 8.-13. März 1999 & 15.-18. März 1999 \\
April 1999 & 5.-10. April 1999 (ausgenommen & 12.-15. April 1999 \\
& Ostermontag am 5. April) & \\
Mai 1999 & $\begin{array}{l}\text { 10.-15. Mai 1999 (ausgenommen } \\
\text { Christi Himmelfahrt am 13. Mai) }\end{array}$ & \\
Juni 1999 & 14.-19. Juni 1999 1999 \\
\hline
\end{tabular}

schließen lassen, dass auch die Rally nicht von der gesamten Wählerschaft getragen wird? Das Design der Inhaltsanalyse ist eng an die aufgeworfenen Fragen angelehnt: Die Wahl von FAZ und SZ als betrachtete Medien bietet neben deren Meinungsführerschaft den weiteren Vorteil, dass die FAZ als gemäßigt rechte und die SZ als gemäßigt linke Zeitung unterschiedliche ideologische Spektren bedienen. Die Darstellung der FAZ wird somit eher die Wähler rechts von der politischen Mitte erreichen, also die CDUund FDP-Anhänger, während die Darstellung der SZ für die Bewertung der Regierung durch die linken Wähler, also die SPD- und Grünen-Anhänger, maßgeblich ist.

\subsection{Präsenz des Kosovokriegs in der Medienberichterstattung}

Innerhalb der Politikteile von FAZ und SZ wurden nur die Artikel ausgewählt, die den Kosovo-Konflikt oder dessen Folgen (mit) zum Thema hatten, dies waren insgesamt 568 Artikel. Tabelle 2 zeigt die Zahl der Artikel nach Monat und Zeitung. Während das Thema Kosovokonflikt vor Beginn der Luftangriffe am 24. März 1999 noch wenig präsent war, ändert sich dies mit Kriegsbeginn. Auffallend ist auch das abnehmende Interesse am Kosovokrieg ab Mai 1999. Die Artikel verteilen sich dabei recht gleichmäßig auf beide Zeitungen. Betrachtet man die Anzahl der Artikel, die auf Seite 1 stehen (in Klammern angegeben), wird die prominente Platzierung des Themas Kosovokonflikt ab April 1999 deutlich. So gibt es ab Kriegsbeginn im untersuchten Zeitraum keinen Tag, an dem nicht auf Seite 1 über den Kosovokrieg zu lesen war. Es ist daher plausibel anzunehmen, dass der Kosovokonflikt ab April 1999 so umfangreich und prominent in den Medien vertreten war, dass er zur Grundlage der Bewertung der Regierungsakteure durch die Wähler werden konnte.

Tabelle 2: Häufigkeit der Artikel zum Kosovokonflikt nach Monat und Zeitung**

\begin{tabular}{lrrr}
\hline & \multicolumn{1}{c}{ FAZ } & \multicolumn{1}{c}{ SZ } & insgesamt \\
\hline 8.-13. März 1999 & $9(2)$ & $10(3)$ & $19(5)$ \\
5.-10. April 1999 & $123(14)$ & $117(16)$ & $240(30)$ \\
10.-15. Mai 1999 & $76(20)$ & $92(11)$ & $168(31)$ \\
14.-19. Juni 1999 & $64(10)$ & $77(8)$ & $141(18)$ \\
insgesamt & $272(46)$ & $296(38)$ & $568(84)$ \\
\hline
\end{tabular}

* Zahl der Artikel auf Seite 1 in Klammern 


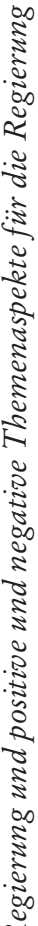

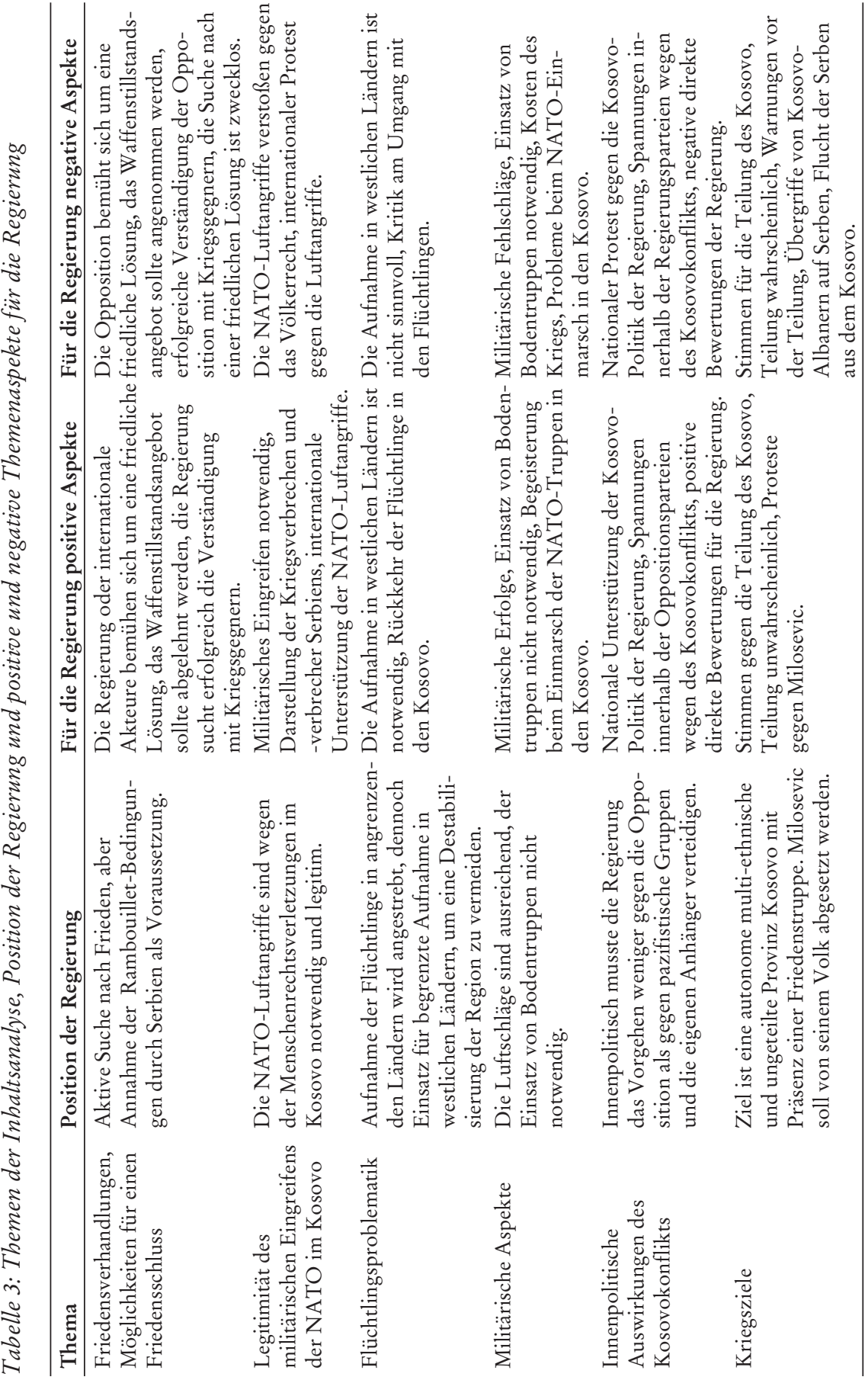




\subsection{Darstellung des Kosovokriegs in den Medien}

Die zweite Anforderung an die Inhaltsanalyse bezieht sich auf die Darstellung des Kosovokriegs in Bezug auf die Regierung. Die Framing-Hypothese, die im zweiten Abschnitt zur Basis der Medienwirkung gemacht wurde, besagt, dass durch das Hervorheben bestimmter Aspekte eines Ereignisses durch die Medien bestimmte Bewertungsgrundlagen bei den Rezipienten aktiviert werden (Scheufele, 2003: 46). Doch welche Aspekte setzen den Kosovokrieg in ein für die Regierung positives Licht und welche sind negativ mit der Regierung verbunden? Da eine Rally in diesem Beitrag damit erklärt wird, dass die Regierung zu Beginn einer internationalen Krise ein Informationsmonopol besitzt und somit ausschließlich ihre Sicht der Dinge in den Medien dargestellt wird, erscheint es sinnvoll anzunehmen, dass die Regierung solche Informationen veröffentlichen wird, die ihre Position zum Konflikt stärken sollen. Dies werden neben direkten Aussagen über die Richtigkeit des Vorgehens der Regierung vor allem Themenaspekte sein, die die Position der Regierung unterstützen. Artikel, die auf den ersten Blick keinerlei Bezug zur Regierung aufweisen, können so dennoch regierungsfreundlich sein, wie z. B. Darstellungen von Kriegsverbrechen an Kosovo-Albanern, die deutlich machen, dass man diese Menschen nicht ihrem Schicksal überlassen darf, was auch der Position der Regierung entspräche. Aus der Betonung verschiedener Themenaspekte und deren Bezug zur Position der Regierung kann man also ablesen, ob die Mediendarstellung eher regierungsfreundlich oder -feindlich ist.

Die 568 Artikel wurden daher auf das Vorkommen von insgesamt 37 Themenaspekten zum Kosovokrieg überprüft, die in Bezug zur Position der Regierung gesetzt wurden und damit positiv oder negativ für die Regierung waren. Zur einfacheren Darstellung wurde diese Vielzahl an Themenaspekten dann in sechs größere kontrovers diskutierte Themen zusammengefasst. Die Analyseebene der Inhaltsanalyse wechselt nun also von den Artikeln zum Kosovokrieg zur Behandlung von Themenaspekten innerhalb dieser Artikel, wobei in einem Artikel mehrere Themenaspekte angesprochen werden konnten. Themen, Position der Regierung und positive und negative Aspekte in Bezug auf diese Position sind Tabelle $3 \mathrm{zu}$ entnehmen.

Innerhalb der Themen wurden Nennungen negativer Themenaspekte von den positiven pro Zeitung und Monat abgezogen. Um dieses Gesamtbild des Kosovokriegs in Bezug auf die Regierungsposition zwischen beiden Zeitungen vergleichbar zu machen, wurden zur Gesamtthemenzahl pro Monat und Zeitung relative Nennungen verwendet. Die Häufigkeit der übergeordneten Themen sollte in erster Linie von aktuellen Ereignissen abhängen und keine Framing-Entscheidungen der Medien widerspiegeln, da sich diese im Gegensatz zu den Themenaspekten noch nicht in Verbindung zur Position der Regierung bringen lassen. Alleine die Betonung unterschiedlicher Themenaspekte sollte daher die Framing-Entscheidungen der Medien ausdrücken. Um dies zu überprüfen und um zu sehen, wie stark die übergeordneten Themen zu unterschiedlichen Zeitpunkten in den Medien vertreten waren, wird in Tabelle 4 abgetragen, wie viel Raum ein Thema pro Monat und Zeitung einnahm.

Die Schaubilder 1 bis 3 stellen den Saldo von relativen Nennungen positiver und negativer Themenaspekte in den beiden Zeitungen zu verschiedenen Themen dar.

Die Schaubilder beschränken sich auf die Themen, die während des Kosovokriegs stark präsent waren, und damit auf die Legitimität des militärischen Eingreifens der NATO, militärische Aspekte und innenpolitische Auswirkungen des Kosovokonflikts. Das Thema der Friedensverhandlungen und die Möglichkeiten für einen Friedensschluss (nicht abgebildet) war hingegen vor dem Beginn des Kosovokriegs, also im März 
Tabelle 4: Präsenz der übergeordneten Themen

\begin{tabular}{|c|c|c|c|c|}
\hline & März & April & Mai & Juni \\
\hline \multirow[t]{2}{*}{ Friedensverhandlungen } & FAZ: $82 \%$, & FAZ: $11 \%$, & FAZ: $18 \%$, & FAZ: $1 \%$, \\
\hline & SZ: $92 \%$ & SZ: $14 \%$ & SZ: $12 \%$ & SZ: $1 \%$ \\
\hline \multirow[t]{2}{*}{ Legitimität des militärischen Eingreifens } & FAZ: $18 \%$, & FAZ: $31 \%$, & FAZ: $29 \%$, & FAZ: $25 \%$, \\
\hline & SZ: $8 \%$ & SZ: $32 \%$ & SZ: $33 \%$ & SZ: $19 \%$ \\
\hline \multirow[t]{2}{*}{ Flüchtlingsproblematik } & FAZ: $0 \%$ & FAZ: $19 \%$, & FAZ: $2 \%$, & FAZ: $12 \%$, \\
\hline & SZ: ०\% & SZ: $16 \%$ & SZ: $9 \%$ & SZ: $11 \%$ \\
\hline \multirow[t]{2}{*}{ Militärische Aspekte } & FAZ: $0 \%$, & FAZ: $24 \%$, & FAZ: $17 \%$, & FAZ: $19 \%$, \\
\hline & SZ: $0 \%$ & SZ: $20 \%$ & SZ: $22 \%$ & SZ: $28 \%$ \\
\hline \multirow[t]{2}{*}{ Innenpolitische Auswirkungen } & FAZ: $0 \%$, & FAZ: $16 \%$, & FAZ: $33 \%$, & FAZ: 7\%, \\
\hline & SZ: $0 \%$ & SZ: $18 \%$ & SZ: $23 \%$ & SZ: 9\% \\
\hline \multirow[t]{2}{*}{ Kriegsziele } & FAZ: 0\%, & FAZ: 0\%, & FAZ: 0\%, & FAZ: $37 \%$, \\
\hline & SZ: ०\% & SZ: 0\% & SZ: 0\% & SZ: $31 \%$ \\
\hline
\end{tabular}

1999, sehr präsent. Die FAZ betont hier positive Themenaspekte, während die SZ stärker auf für die Regierung negative Aspekte eingeht. Dies sollte für die Bewertung der Regierung aufgrund der geringen Präsenz des Kosovokriegs zu diesem Zeitpunkt jedoch wenig relevant sein.

Vor der Besprechung der Schaubilder 1 bis 3 folgt zur besseren Verständlichkeit ein Beispiel zum Zustandekommen des Saldos positiver und negativer Themenaspekte: Die FAZ hat im April in 123 Artikeln 191 Themenaspekte angesprochen. 39 dieser Aspekte gehören zum Thema Legitimität des militärischen Eingreifens der NATO im Kosovo und decken sich mit der Position der Bundesregierung. Relativ gesehen sind also 20,4 \% der in der FAZ im April angesprochenen Themenaspekte positiv für die Regierung. Dagegen wurden nur 14 negative Aspekte für diesen Themenbereich genannt, also 7,3 \%.

Schaubild 1: Saldo negativer und positiver Themenaspekte zum Thema Legitimität des militärischen Eingreifens der NATO im Kosovo

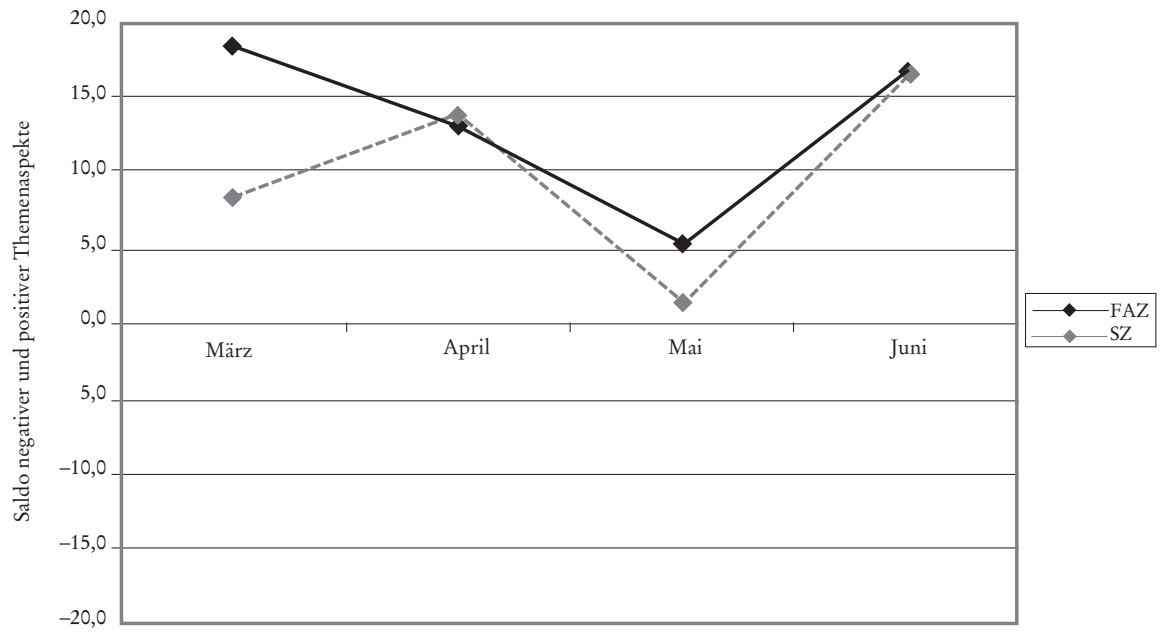


Schaubild 2: Saldo negativer und positiver Themenaspekte zum Thema Militärische Aspekte

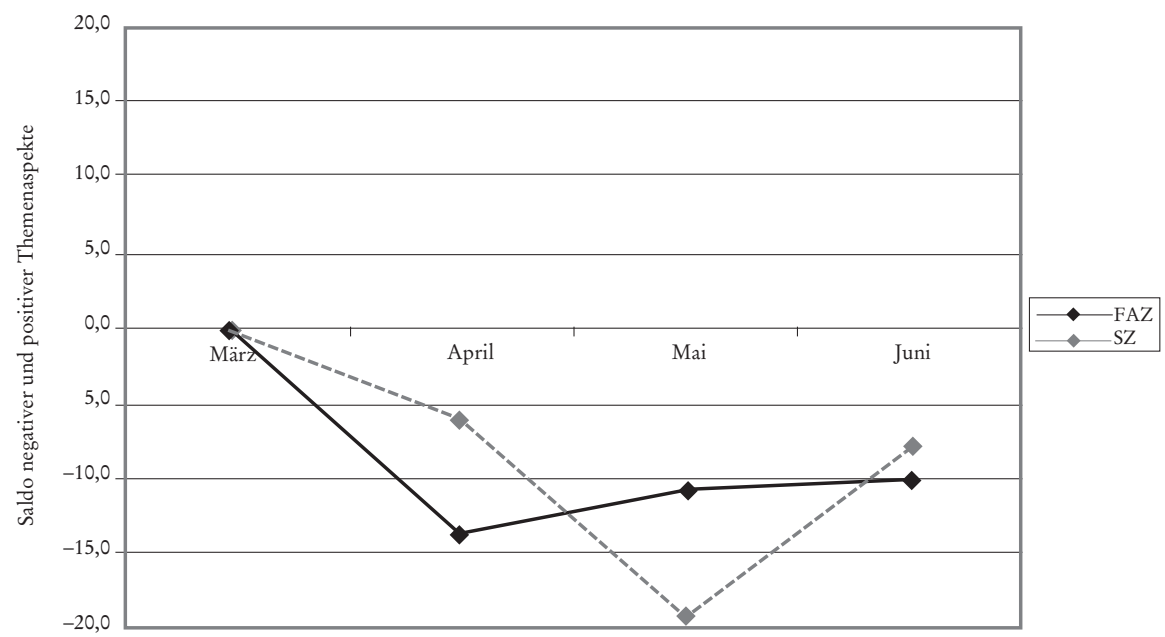

Der Saldo der genannten positiven und negativen Themenaspekte beträgt also 13,1 \%, diese Zahl findet sich in Schaubild 1 für die FAZ im April wieder.

Das Thema Legitimität des militärischen Eingreifens der NATO im Kosovo ist das am kontinuierlichsten in den Medien vertretene. Erstaunlich ist die sehr positive Darstellung dieses Themas für die Regierung im April sowohl in der FAZ als auch in der SZ. Mit jeweils gut $30 \%$ der Nennungen ist dieses Thema auch das wichtigste nach Kriegs-

Schaubild 3: Saldo negativer und positiver Themenaspekte zum Thema Innenpolitische Auswirkungen des Kosovokonflikts

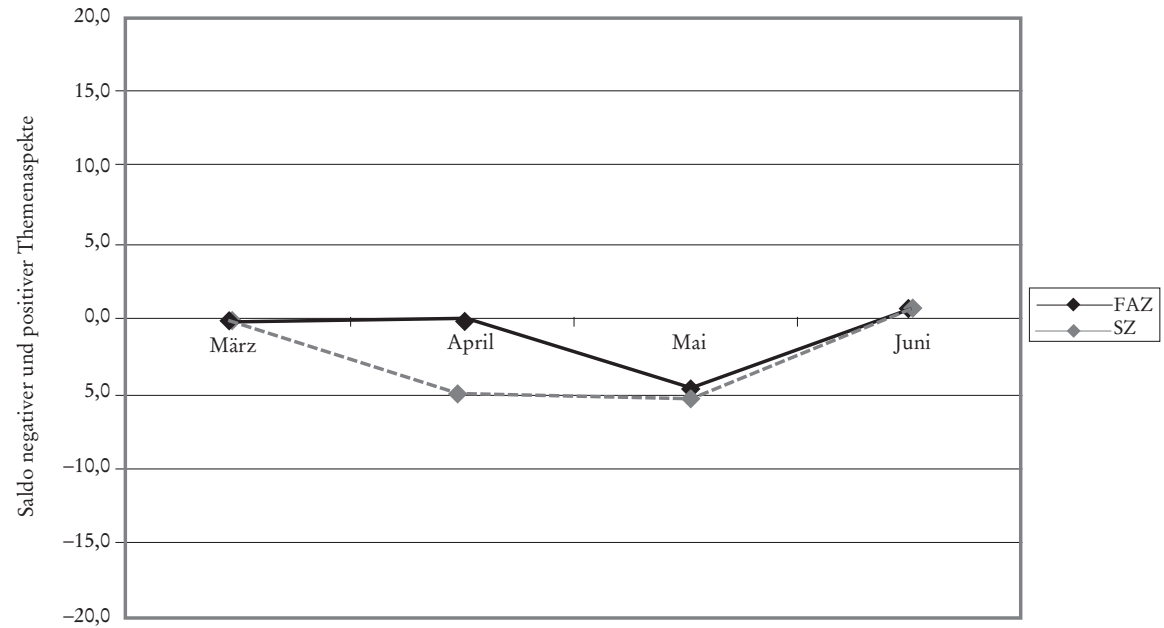


beginn. Im Mai hingegen wird die Legitimität des Krieges in beiden Zeitungen stärker angezweifelt, um im Juni durch die Entdeckungen von Massakern und Massengräbern wieder gegeben zu sein.

Ganz anders stellt sich der Themenbereich Militärische Aspekte dar. Schon im April werden überwiegend negative Themenaspekte herausgestellt, von der FAZ noch stärker als von der SZ. Diese reagiert im Mai sehr sensibel auf die große Zahl an Kollateralschäden, was das Bild des Kosovokriegs in der SZ sehr negativ werden lässt, um sich im Juni, mit dem gewonnenen Krieg, stark für die Regierung zu verbessern. Die FAZ bleibt in der Nennung negativer Themenaspekte dagegen konstant hoch und relativ stabil.

Da der Kosovokrieg innerhalb Deutschlands stark umstritten war, wird auch das Thema der innenpolitischen Auswirkungen in für die Regierung negativen Aspekten dargestellt, im April schon von der SZ, im Mai folgt auch die FAZ. Erst im Juni wird die Berichterstattung positiver für die Regierung, dass Thema spielt aber nur noch eine geringe Rolle.

Im Gegensatz dazu gewinnt das Thema Flüchtlingsproblematik (nicht abgebildet) im Juni erneut an Brisanz und wird von beiden Zeitungen für die Regierung positiv dargestellt. Schon kurz nach Kriegsbeginn, im April, spielte dieses Thema eine Rolle, die Berichterstattung war in beiden Zeitungen jedoch recht neutral, so dass sich hieraus keine Schlüsse auf die Bewertung der Regierung ziehen lassen.

Das Thema Kriegsziele wurde durch die Diskussion, ob die Kriegsziele erreicht wurden, erst nach dem Krieg, im Juni, präsent und nimmt dann mit $37 \%$ bei der FAZ und $31 \%$ bei der SZ auch viel Raum ein. Beide Zeitungen machen durch die Betonung negativer Themenaspekte dabei deutlich, dass die Ziele nicht erreicht wurden, wobei die SZ noch negativer als die FAZ urteilt.

Um einen Überblick über die Darstellung des Kosovokriegs in Relation zur Regierungsposition über die vorgestellten Themen hinweg zu bekommen, fasst Tabelle 5 die Befunde der Inhaltsanalyse zusammen. Hierfür wurden die in den Schaubildern abgetragenen positiven oder negativen Darstellungen der verschiedenen Themen zusammengezählt und anhand der Themenpräsenz pro Monat, wie in Tabelle 4 angegeben, gewichtet, wodurch man zu einer Gesamtdarstellung des Kosovokriegs in Bezug auf die Regierung in FAZ und SZ gelangt.

Durch die hohe Präsenz des Themas Legitimität des militärischen Eingreifens der NATO im Kosovo und hierbei der Betonung von Themenaspekten, die die Position der Regierung stützen, ist die Medienberichterstattung trotz der negativen Darstellung des Themas Militärische Aspekte in beiden Zeitungen im April eher regierungsfreundlich. Doch schon im Mai wird das Medienbild des Kosovokriegs für die Regierung durch die hohe Zahl an Kollateralschäden und die innenpolitischen Legitimationsprobleme der Regierung in der SZ negativer, während die FAZ noch ein für die Regierung positives Bild vermittelt. Dies ändert sich im Juni mit dem Aufkommen der Frage, ob die Kriegs-

Tabelle 5: Nach Themenpräsenz gewichtete Gesamtdarstellung des Kosovokriegs

\begin{tabular}{lll}
\hline & FAZ & SZ \\
\hline März & 10,7 & $-6,9$ \\
April & 1,6 & 2,5 \\
Mai & 2,5 & -4 \\
Juni & -2 & $-3,4$ \\
\hline
\end{tabular}


ziele erreicht wurden. Dieses Thema wird von beiden Zeitungen prominent und in für die Regierung negativen Themenaspekten dargestellt, so dass die Berichterstattung zum Kosovokrieg im Juni eher regierungsfeindlich war.

\subsection{Hypothesen zur Wirkung des Kosovokriegs auf die Popularität der Regierungsak- teure}

Da die für Priming-Effekte notwendige Bedingung der starken Präsenz des Themas in der Medienberichterstattung für den Kosovokrieg erfüllt war, kann man davon ausgehen, dass der Kosovokrieg zur Bewertungsgrundlage der Wähler für die politischen Akteure wurde. Hinsichtlich der Richtung einer Bewertungsänderung durch Framing-Effekte muss man im Anschluss an die theoretische Diskussion zwei Dimensionen unterscheiden: zum einen, welchen politischen Akteuren eine mögliche Rally zugute kommt, und zum anderen, welche Teile der Wählerschaft diese Rally tragen. Für Bündnis 90/Die Grünen als kleinem Koalitionspartner kann man vermuten, dass sie aufgrund ihres geringeren Handlungsspielraums in der Koalition und ihrer geringeren Sichtbarkeit einerseits weniger stark von Popularitätsveränderungen durch den Kosovokrieg betroffen sein werden. Andererseits aber wirkt diese Partei nach der Bundestagswahl 1998 zum ersten Mal an einer Bundesregierung mit, der Kosovokrieg stellt für sie also einen Prüfstein dahingehend dar, wie sie sich bei einem Konflikt zwischen ideologischen Grundlagen und internationalen Verpflichtungen verhalten wird. Da die Grünen somit unter besonderer Beobachtung durch die Wähler stehen, sollten sowohl die Partei als auch ihre Politiker von positiven wie negativen Entwicklungen stärker als die SPD betroffen sein. Die Frage nach unterschiedlichen Rally-Effekten zwischen verschiedenen Wählergruppen wird anhand der Berichterstattung der SZ als Medium eher linker Gruppen, also den Anhängern von SPD und Bündnis 90/Die Grünen, und der FAZ als gemäßigt rechter Zeitung und damit dem Medium der CDU- und FDP-Anhänger, beantwortet. Die Hypothesen lauten wie folgt:

Hypothese 1: Im April sollte die Popularität aller Regierungsakteure aufgrund des Vorherrschens von für die Regierung vorteilhaften Themen steigen, dabei die von Bündnis 90/Die Grünen und Außenminister Fischer stärker als die der SPD, Schröders und Scharpings. Dieser Anstieg sollte von allen Teilen der Wählerschaft gleichermaßen getragen werden.

Hypothese 2: Die Popularität der Regierungsakteure sollte dagegen schon im Mai sinken, die von Bündnis 90/Die Grünen und Fischer stärker als die der SPD und von deren Politikern. Dieser Abschwung wird in erster Linie von den Anhängern der SPD und Bündnis 90/Die Grünen verursacht, da sie stärker mit für die Regierung negativen Informationen versorgt werden. Die CDU- und FDP-Anhänger sollten die Regierung aufgrund der weniger negativen Darstellung der FAZ weiterhin unterstützen, so dass deren Popularität im Mai noch recht hoch sein wird. Die Unabhängigen werden in ihrem Entzug an Unterstützung zwischen Regierungs- und Oppositionsanhängern liegen.

Hypothese 3: Die Distanzierung der SPD- und Grünen-Anhänger von der Regierung sollte sich im Juni fortsetzen. Im Gegensatz zum Mai distanzieren sich nun aber auch andere Teile der Wählerschaft, so dass die Popularität der Regierung im Juni drastisch sinken wird, allen voran die Popularität von Bündnis 90/Die Grünen und von Fischer. 


\section{Konstruktion der Regierungspopularität aus Umfragedaten und Ergebnisse}

\subsection{Methode}

Eine Möglichkeit zur Messung der Regierungspopularität in Umfragen stellt die so genannte Skalometerfrage dar. Hierbei werden die Befragten gebeten, die Parteien und Politiker aufgrund ihrer Sympathie auf einer Skala von -5 bis 5 einzustufen. ${ }^{3}$ Der Vorteil der Skalometerfrage besteht darin, dass sie nicht auf ein bestimmtes Thema oder eine bestimmte Eigenschaft der politischen Akteure zielt, die zur Basis der Bewertung herangezogen werden soll, sondern ein allgemeines Urteil fordert. In den Einstufungen der Befragten werden sich daher zum einen die ideologische Nähe politischer Akteure und zum anderen davon unabhängige Beurteilungen widerspiegeln (Enelow/Hinich, 1984: 170). Aufgabe ist es nun, aus den beobachteten Skalometerwerten der Befragten die zugrundeliegenden latenten Dimensionen zu extrahieren, also die ideologische Bewertung von der Popularität zu trennen. Hierfür wird eine explorative Faktorenanalyse durchgeführt. Die Faktoren werden hierbei anhand des Hauptkomponentenverfahrens extrahiert, wodurch die Varianz, die die Faktoren erklären, maximiert wird (Revenstorf, 1980: 71ff.). Die Basis der Faktorenanalyse bildet die Varianz-Kovarianz-Matrix der Skalometerwerte der Befragten zu den politischen Akteuren. Als Bezugspunkt wurde der Nullpunkt verwendet, da dieser von der Skalometerskala vorgegeben wird und damit eine fehlerfreie Distanzmessung zwischen den Parteien gewährleistet (vgl. zur Methode den Anhang in Pappi u. a., 2004). Zur Extrahierung der relevanten Bewertungsdimensionen und der Anordnung politischer Akteure in einem durch die Bewertungsdimensionen aufgespannten politischen Raum durch eine Faktorenanalyse sind die Beziehungen dieser Akteure untereinander wesentlich, weshalb die Analyse neben den genannten Regierungsparteien und -politikern auch CDU, CSU, FDP, PDS und die Parteivorsitzenden Schäuble und Stoiber umfasst. Die Faktorladungen stellen dabei die Positionen der Parteien und Politiker im politischen Raum dar. Die Validierung der Bedeutung der X-Achse dieses Raums als ideologische und der Y-Achse als Popularitätsdimension geschieht über externe Daten. ${ }^{4}$ Zusätzlich zur Trennung der Skalometerwerte in ideologische und Popularitätsdimension und der Konstruierung eines politischen

3 Der genaue Fragentext lautet: „Stellen Sie sich einmal ein Thermometer vor, das aber lediglich von plus 5 bis minus 5 geht, mit einem Nullpunkt dazwischen. Sagen Sie es bitte mit diesem Thermometer, was Sie von den einzelnen Parteien halten. +5 bedeutet, dass Sie sehr viel von der Partei halten. -5 bedeutet, dass Sie überhaupt nichts von der Partei halten. Mit den Werten dazwischen können Sie Ihre Meinung abgestuft sagen.“

4 Hierbei werden zum einen die Ladungen der Parteien auf der ersten Dimension auf die ideologischen Einstufungen der Parteien durch die Wähler rotiert. Hinsichtlich der Rotation der Ladungen auf der zweiten Dimension kann aufgrund des Mangels an Popularitätsdaten nur der zweitbeste Weg beschritten werden, nämlich die Rotation auf externe Skalometerdaten, die wenigstens zum Teil die Popularität der Parteien widerspiegeln. Es wird daher erwartet, dass die Korrelation der zweiten Dimension mit den Skalometerfragen geringer ausfällt als die der ersten Dimension mit den ideologischen Einstufungen. Die hierfür herangezogenen Daten sind die Nachwahldaten zur Bundestagswahl 1998 des DFG-Projekts „Politische Einstellungen, politische Partizipation und Wählerverhalten im vereinigten Deutschland“ (ZA-Nr. S3064). Erwartungsgemäß korrelieren die Positionen der Parteien auf der X-Achse hoch mit der LinksRechts-Einstufung (Korrelationskoeffizient zwischen 0,90 und 0,95 in Westdeutschland), während die Positionen auf der Y-Achse mit der Skalometereinstufung korreliert sind (Korrelationskoeffizienten zwischen 0,78 und 0,91 in Westdeutschland). 
Raums lassen sich mit der gewählten Methode auch die Befragten über die Faktorwerte in diesem Raum darstellen, also den für sie idealen Punkt ermitteln. Hinsichtlich der ideologischen Dimension kann dieser Punkt irgendwo im politischen Raum liegen und gibt die ideologische Nähe des Befragten zu den Parteien und Politikern an. Dies trifft jedoch nicht auf die Popularitätsdimension zu, da hier bekannt ist, dass die Position aller Befragten gleich dem höchsten bei der Skalometerfrage zu vergebenden Wert, also der Fünf, ist, der die meist präferierte Partei ausdrückt. Daher werden die Faktorwerte der Befragten auf der zweiten Dimension um den Wert 5 transformiert (vgl. zur Methode den Anhang in Pappi u. a., 2004). Die Idealpunkte der Befragten werden anhand von Parteianhängerschaften zusammengefasst, so dass der politische Raum neben den genannten Parteien und Politikern die Mediane der Idealpunkte für die Anhänger von SPD, Bündnis 90/Die Grünen, der Union, FDP und der Unabhängigen enthält. Die Anhängerschaft zu einer Partei wird über die in Deutschland gebräuchliche Parteineigerfrage festgestellt. ${ }^{5}$ Auf der Popularitätsdimension niedrige Werte bestimmter Anhängergruppen bedeuten dabei nicht, dass diese Befragten eine inkompetente Partei präferieren, sondern dass es nur relativ wenig Befragte außer ihnen gibt, für die diese Partei sehr populär ist, da die Faktorenanalyse die politischen Beziehungen aus der Sicht des Durchschnittsbefragten wiedergibt. Die Faktorenanalyse wird für Ost- und Westdeutschland wegen der unterschiedlichen Parteiensysteme getrennt durchgeführt. Im Folgenden werden jedoch nur die Ergebnisse für Westdeutschland abgebildet, die Ergebnisse für Ostdeutschland werden im Text besprochen, soweit sie von den westdeutschen abweichen.

\subsection{Ergebnisse}

Popularitätsveränderungen von Parteien und Politikern drücken sich durch Veränderungen der Positionen auf der Y-Achse, also der Popularitätsdimension, aus. Durch welche Teile der Wählerschaft diese Veränderung zustande kommt, wer also eine mögliche Rally trägt, kann über die Veränderung der Distanzen zwischen politischen Akteuren und verschiedenen Anhängerschaften ermittelt werden.

Die Ausgangskonstellation im März, in Schaubild 4 dargestellt, zeigt deutlich die ideologische Links-Rechts-Anordnung der politischen Akteure auf der X-Achse und die um ihre Parteien gruppierten Anhänger mit den Unabhängigen relativ in der Mitte mit Tendenz zum rechten Lager. Erstaunlich ist, dass Fischer schon im März sowohl ideologisch als auch in seiner Popularität recht nah an der SPD und Schröder positioniert und damit weit entfernt von seiner Partei ist.

Zusätzlich zu den Positionen der politischen Akteure und Anhängerschaften im aktuellen Monat werden in den folgenden Schaubildern die Positionen der Regierungsakteure und der Anhängerschaften im Vormonat (graue Rauten und Quadrate) abgetragen, um Veränderungen deutlich zu machen. Wie in Hypothese 1 konstatiert wurde und Schaubild $5 \mathrm{zu}$ entnehmen ist, steigen die Regierungsakteure in ihrer Popularität, dargestellt durch die Bewegungen auf der Y-Achse. Am stärksten profitiert Verteidigungsminister Scharping von diesem Aufwärtstrend. Entgegen der Erwartungen können Bündnis 90/Die Grünen jedoch nicht von diesem Popularitätsschub profitieren. Die

5 Der genaue Wortlaut der Parteineigerfrage: „In Deutschland neigen viele Leute über eine längere Zeit einer bestimmten Partei zu, obwohl sie ab und zu auch eine andere Partei wählen. Wie ist das bei Ihnen: Neigen Sie - ganz allgemein gesprochen - einer bestimmten Partei zu? Wenn ja, welcher?" 
Schaubild 4: Positionen der Parteien und Politiker und Median der Idealpunkte nach Anhängergruppen im März 1999 in Westdeutschland*

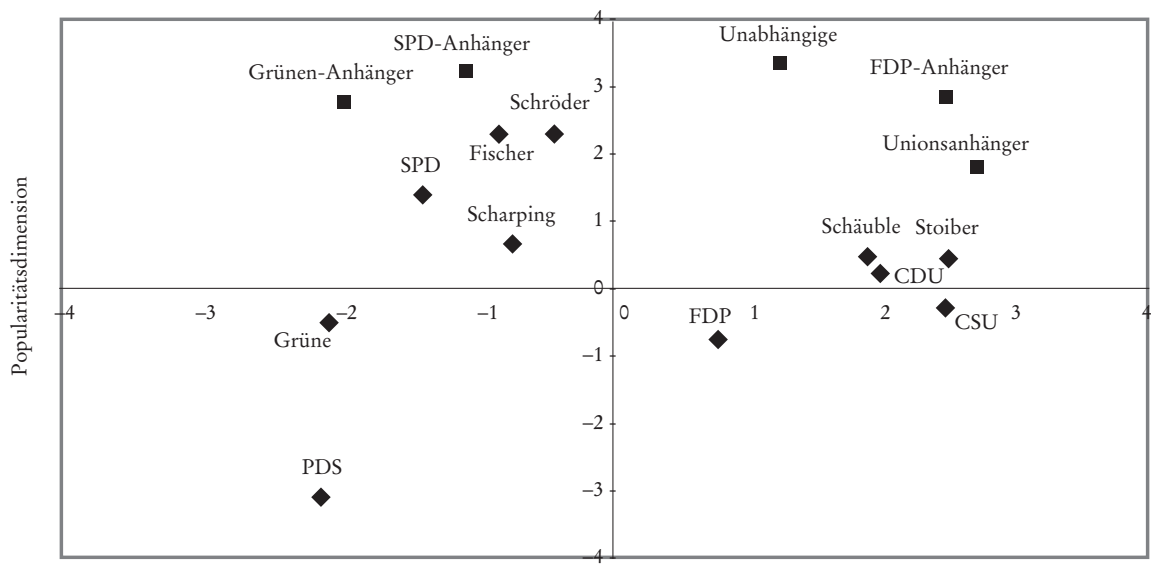

Ideologische Dimension

* Die erklärte Varianz des ersten Faktors (ideologische Dimension) beträgt 35 \%, die des zweiten (Popularitätsdimension) $23 \%$.

Schaubild 5: Positionen der Parteien und Politiker und Median der Idealpunkte nach Anhängergruppen im April 1999 in Westdeutschland**

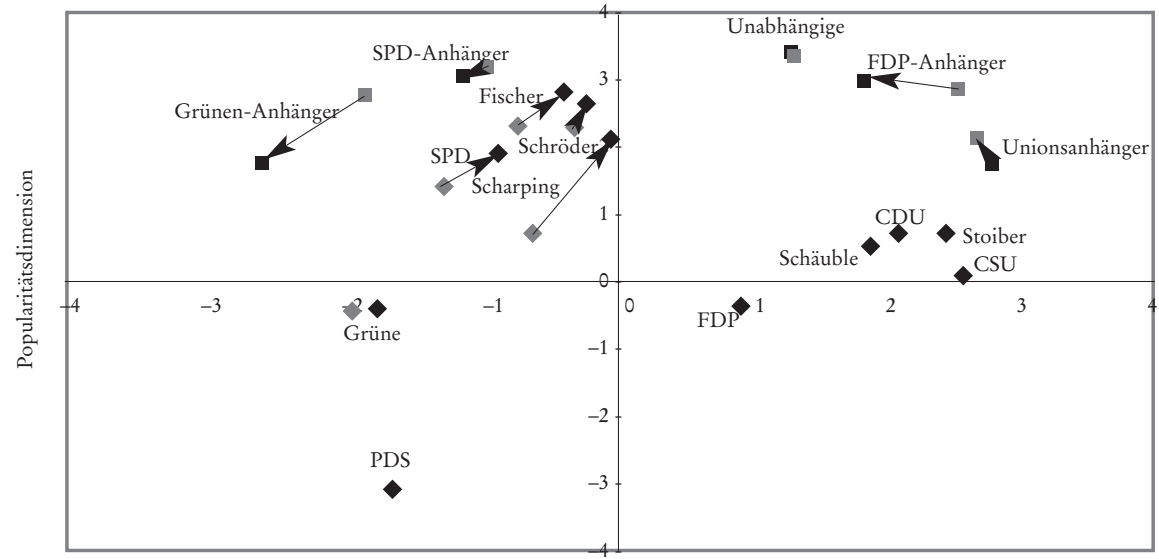

Ideologische Dimension

* Die erklärte Varianz des ersten Faktors (ideologische Dimension) beträgt $43 \%$, die des zweiten (Popularitätsdimension) $24 \%$.

Rally zugunsten der anderen Regierungsakteure scheint von den Anhängern aller Parteien gleichmäßig getragen worden zu sein, es hebt sich nur die kleine Zahl an FDP-Anhängern durch ein Näherrücken an das Regierungslager hervor. 
Im Unterschied zu Westdeutschland können jedoch im Osten (nicht dargestellt) auch Bündnis 90/Die Grünen von der Rally profitieren, ihre eigenen Anhänger gehen aber, wie auch im Westen, auf Distanz zu ihrer Partei. Die Rally wird im Gegensatz zu Westdeutschland in erster Linie von den Unabhängigen getragen.

Im Mai sinken die Regierungsakteure wie vorhergesagt etwas in ihrer Popularität ab, von diesem Abschwung sind am stärksten Bündnis 90/Die Grünen betroffen, wie Schaubild $6 \mathrm{zu}$ entnehmen ist. Sie profitieren als Antikriegspartei also nicht von der Rally, leiden aber unter dem Entzug der Unterstützung durch die schon im zweiten Kriegsmonat zum Teil stark negative Berichterstattung. Entgegen der zweiten Hypothese, wonach die weiterhin hohe Popularität der Regierung im Mai stärker von Anhängern des rechten Lagers und der Unabhängigen getragen werden sollte, distanzieren sich FDPAnhänger und Unabhängige im Mai vom Regierungslager, während die Grünen-Anhänger wieder näher an ihre Partei rücken. Diese Bewegung der Grünen-Anhänger tritt in Ostdeutschland nicht auf (nicht dargestellt).

\section{Schaubild 6: Positionen der Parteien und Politiker und Median der Idealpunkte nach} Anbängergruppen im Mai 1999 in Westdeutschland*

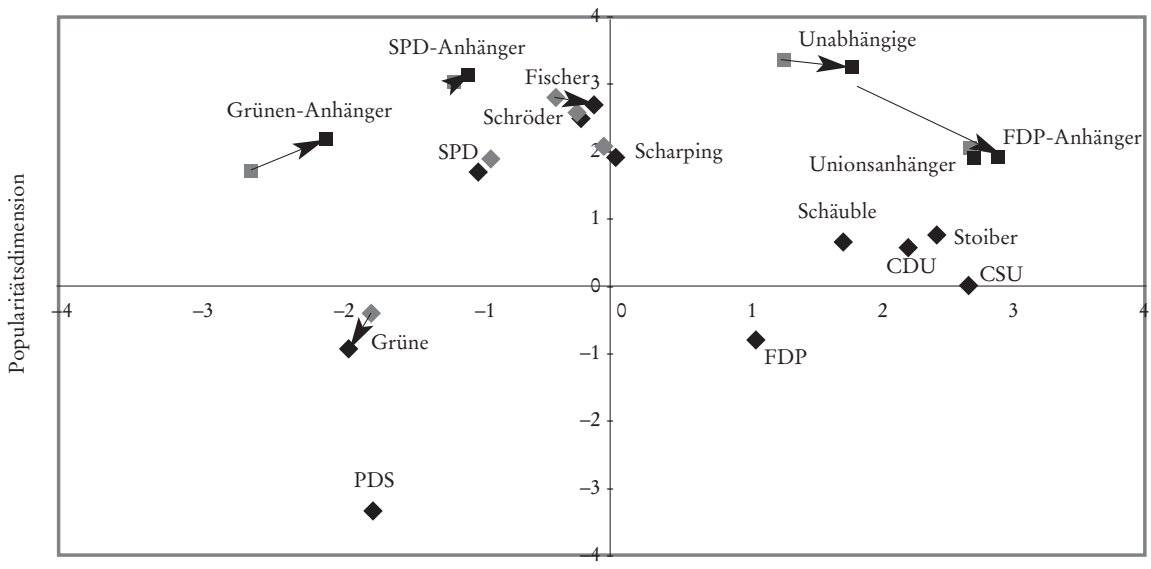

Ideologische Dimension

\footnotetext{
* Die erklärte Varianz des ersten Faktors (ideologische Dimension) beträgt 44 \%, die des zweiten (Popularitätsdimension) $23 \%$.
}

Wie in Schaubild 7 zu sehen ist, sinkt die Popularität von SPD, Schröder und Scharping im Juni erwartungsgemäß weiter. Auch die Unabhängigen und FDP-Anhänger nähern sich wieder der Regierung an. Nach dem Krieg scheint sich die Situation wieder zu normalisieren. Fischer und Scharping bleiben hingegen sehr populär, haben sich in der Wahrnehmung der Wähler also durch den Kosovokrieg von Parteipolitikern zu Staatsmännern gewandelt.

Zusammenfassend kann zur Entwicklung in Westdeutschland gesagt werden, dass im April, direkt nach Kriegsbeginn, tatsächlich eine Rally stattfindet, wie von der ersten Hypothese auch konstatiert wurde. Entgegen den Erwartungen können Bündnis 90/Die Grünen als pazifistische Partei jedoch nicht von der Rally profitieren. Erwartungsgemäß dauert dieser Popularitätsanstieg nicht lange an, schon im Mai distanzieren sich etliche 
Schaubild 7: Positionen der Parteien und Politiker und Median der Idealpunkte nach Anbängergruppen im Juni 1999 in Westdeutschland**

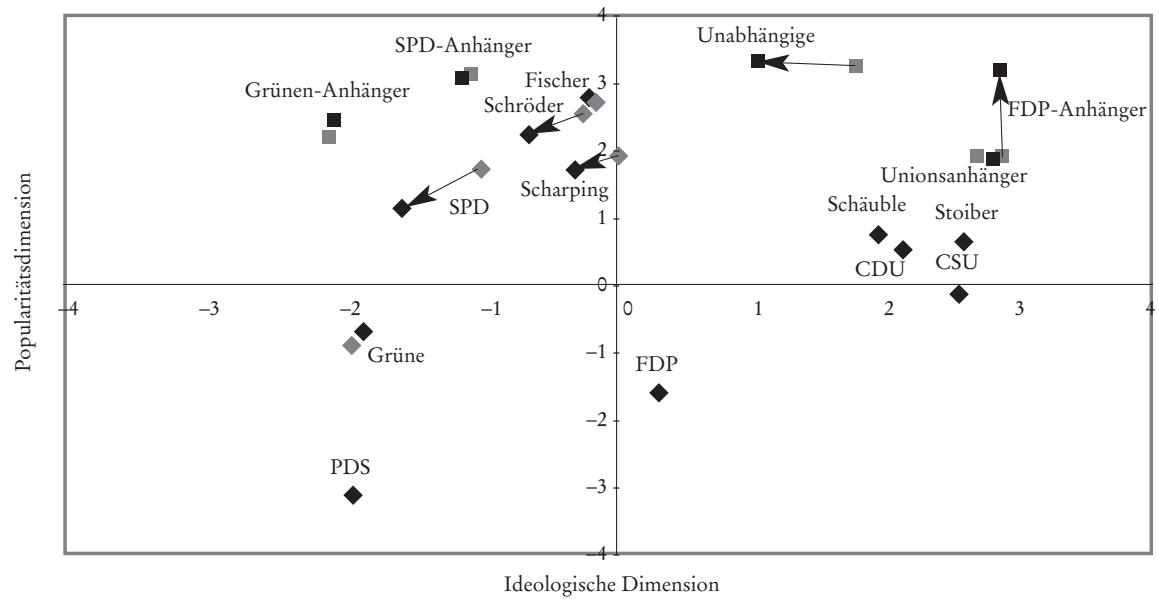
* Die erklärte Varianz des ersten Faktors (ideologische Dimension) beträgt $41 \%$, die des zweiten (Popularitäts-
dimension) $25 \%$.

Wähler von den Regierungsakteuren. Entgegen der zweiten Hypothese distanzieren sich jedoch FDP-Anhänger und Unabhängige vom Regierungslager, die hohe Popularität der Regierungsakteure im Mai wird also in erster Linie von den Anhängern der Regierung getragen. Im Juni kann man von einem Ende der Wirkung des Kosovokriegs auf die Popularität der Regierungsparteien und deren Politiker sprechen, bis auf die hohe Popularität Fischers und Scharpings ähnelt das Bild wieder stark der Ausgangskonstellation vor dem Kosovokrieg.

Die Entwicklung in Ostdeutschland ist der westdeutschen trotz der oft betonten kritischeren Haltung der ostdeutschen Bevölkerung zum Kosovokrieg sehr ähnlich. Im Gegensatz zum Westen sind Bündnis 90/Die Grünen in Ostdeutschland jedoch in diese Dynamik eingebunden und profitieren auch von der Rally. Die Entwicklung in Ostdeutschland scheint dabei stärker von der Gruppe der Unabhängigen getragen zu werden als in Westdeutschland.

\section{Ausblick}

Der vorliegende Beitrag geht auf die Möglichkeiten einer Rally, also eines Anstiegs der Regierungspopularität durch einen Krieg, ein und zieht hierfür den Kosovokrieg 1999 heran. Es konnte gezeigt werden, dass durch die hohe Präsenz des Themas Krieg und die Darstellung dessen in für die Regierung positiven Themenaspekten im ersten Kriegsmonat, dem April 1999, eine Rally stattgefunden hat. Hierbei gibt es jedoch Einschränkungen: So stieg die Popularität von Bündnis 90/Die Grünen durch den Kosovokrieg bei großen Teilen der Bevölkerung nicht an. Zudem distanzierten sich die Grünen-Anhänger vom Regierungslager. Als Grund hierfür kann man sich die pazifistische Grundposition von Bündnis 90/Die Grünen und damit auch ihrer Anhänger denken. Dies deutet daraufhin, dass in parlamentarischen Systemen mit Koalitionsregierungen eine Rally auch von den politischen Positionen der Regierungsparteien abhängt, während dies 
für den amerikanischen Präsidenten nicht der Fall zu sein scheint. So scheint eine Rally kaum möglich zu sein, wenn das außenpolitische Verhalten einer Regierungspartei ihren grundlegenden ideologischen Positionen widerspricht.

Ein weiteres wesentliches Ergebnis lautet, dass die Unterstützung entgegen der - zugegebenermaßen nur gering - regierungsfreundlicheren Berichterstattung in der FAZ im April in erster Linie bei den FDP-Anhängern und den Unabhängigen sank, während die Unterstützung der SPD- und Grünen-Anhänger für eine hohe Popularität der Regierungsakteure sorgte. Hieraus kann man folgern, dass die Parteineigung als Basis der Unterstützung für die Regierung durch eine Rally durch eine konsonant regierungsfreundliche Berichterstattung scheinbar kurzzeitig ausgehebelt werden kann, aber mit Dauer des Kriegs und dem Auftreten regierungskritischer Informationen wieder stark an Einfluss auf die Regierungspopularität gewinnt.

Generell muss natürlich gesagt werden, dass anhand eines Fallbeispiels kaum verallgemeinerbare Aussagen über die Wirkungen von Kriegen, oder, wie es der Rally-Begriff vorsieht, von internationalen Krisen, auf die Popularität deutscher Regierungsakteure getroffen werden können. Daher wäre es zum einen erstrebenswert, das Kriegsengagement der Verweigerung der Kriegsteilnahme, wie z. B. beim Irakkrieg 2003, gegenüberzustellen und Auswirkungen in Abhängigkeit von den Positionen der Wählerschaft und der Rechtfertigung des Kriegs zu modellieren. Zum anderen wäre es sinnvoll, eine Vielzahl internationaler Krisen zu sammeln, die eine ähnliche Wirkung wie der Kosovokrieg entfalten könnten, und so über den Einzelfall Kosovokrieg, aber auch über die statistischen Möglichkeiten des vorliegenden Beitrags hinauszugehen. Hier konnte vor allen Dingen gezeigt werden, dass sich die Suche nach Auswirkungen von Kriegen auf die Popularität der deutschen Regierung lohnen kann.

\section{Literatur}

Allen, Barbara; O’Loughlin, Paula; Jasperson, Amy und Sullivan, John L. (1994): The Media and the Gulf War: Framing, Priming, and the Spiral of Silence. In: Polity, 27, S. 255-284.

Brewer, Paul; Aday, Sean und Gross, Kimberly (2003): Rallies All Around: The Dynamics of System Support. In: Pippa Norris, Montague Kern und Marion Just (Hg.): Framing Terrorism. The News Media, the Government and the Public. New York/London, S. 229-253.

Brody, Richard A. (1991): Assessing the President. The Media, Elite Opinion, and Public Support. Stanford.

Callaghan, Karen J. und Virtanen, Simo (1993): Revised Models of the "Rally Phenomenon": The Case of the Carter Presidency. In: The Journal of Politics, 55, S. 756-764.

Donsbach, Wolfgang (1989): Selektive Zuwendung zu Medieninhalten. Einflussfaktoren auf die Auswahlentscheidung der Rezipienten. In: Max Kaase und Winfried Schulz (Hg.): Massenkommunikation. Theorien, Methoden, Befunde. Opladen, S. 392-405.

Enelow, James M. und Hinich, Melvin J. (1984): The Spatial Theory of Voting. An Introduction. Cambridge.

Iyengar, Shanto und Simon, Adam (1993): News Coverage of the Gulf Crisis and Public Opinion. A Study of Agenda-Setting, Priming, and Framing. In: Communication Research, 20, S. 365383.

Kepplinger, Hans Mathias; Donsbach, Wolfgang; Brosius, Hans-Bernd und Staab, Joachim Friedrich (1986): Medientenor und Bevölkerungsmeinung. Eine empirische Studie zum Image Helmut Kohls. In: Kölner Zeitschrift für Soziologie und Sozialpsychologie, 38, S. 247-279.

McLeod, Douglas M.; Eveland, William P. Jr. und Signorielli, Nancy (1994): Conflict and Public Opinion: Rallying Effects of the Persian Gulf War. In: Journalism Quarterly, 71, S. 20-31.

Mueller, John E. (1970): Presidential Popularity from Truman to Johnson. In: American Political Science Review, 64, S. 18-34. 
Mueller, John E. (1973): War, Presidents and Public Opinion. New York.

Norpoth, Helmuth (1988): Krieg und Regierungspopularität: Der Fall der Falklands. In: Friedhelm Meier (Hg.): Prozessforschung in den Sozialwissenschaften. Anwendungen zeitreihenanalytischer Methoden. Stuttgart, S. 1-22.

Pappi, Franz U.; Shikano, Susumu und Bytzek, Evelyn (2004): Der Einfluss politischer Ereignisse auf die Popularität von Parteien und Politikern und auf das Parteiensystem. In: Kölner Zeitschrift für Soziologie und Sozialpsychologie, 56, S. 51-70.

Peter, Jochen (2002): Medien-Priming - Grundlagen, Befunde und Forschungstendenzen. In: Publizistik, 47, S. 21-44.

Revenstorf, Dirk (1980): Faktorenanalyse. Stuttgart u.a.

Schenk, Michael (2002). Medienwirkungsforschung. 2., vollst. überarb. Aufl., Tübingen, S. 296303, S.710

Scheufele, Bertram (2003): Frames - Framing - Framing-Effekte. Theoretische und methodische Grundlegung des Framing-Ansatzes sowie empirische Befunde zur Nachrichtenproduktion. Wiesbaden.

Schütt, Ernst Christian (2000): Chronik 1999. Tag für Tag in Wort und Bild. Gütersloh/ München. Sigelman, Lee und Conover, Pamela Johnston (1981): The Dynamics of Presidential Support during International Crisis: The Iranian Hostage Crisis. In: Political Behavior, 3, S. 303-318. 